\title{
Gossez's skew linear map and its pathological maximally monotone multifunctions
}

\author{
Stephen Simons *
}

\begin{abstract}
In this note, we give a generalization of Gossez's example of a maximally monotone multifunction such that the closure of its range is not convex, using more elementary techniques than in Gossez's original papers. We also discuss some new properties of Gossez's skew linear operator and its adjoint.
\end{abstract}

2010 Mathematics Subject Classification: Primary 47H05; Secondary 47N10, 46A20, 46A22.

Keywords: Skew linear operator, maximal monotonicity, duality map.

\section{Introduction}

In [4] and [5], Gossez gives an example of a skew linear map $G: \ell_{1} \rightarrow \ell_{\infty}=\ell_{1}^{*}$, and proves that there exist arbitrarily small values of $\lambda>0$ such that $\overline{R(G+\lambda J)}$ is not convex. (If $E$ is a Banach space, $J: E \rightrightarrows E^{*}$ is the duality map, defined by $x^{*} \in J x$ exactly when $\left\|x^{*}\right\|=\|x\|$ and $\left\langle x, x^{*}\right\rangle=\|x\|^{2}$. See eqn. (2.4).) In Theorem 3.5, we shall prove the stronger result that $\overline{R(G+\lambda J)}$ is not convex whenever $0<\lambda<4$. In particular, $\overline{R(G+J)}$ is not convex. $(R(\cdot)$ stands for "range of".)

Gossez's analysis goes by way of the monotone extension to the dual introduced in 3. This was critical to his definition of operators of dense type, which have been so important in the modern theory of monotone multifunctions. In addition to the use of the monotone extension to the dual, [4] and [5] use measure theory on the Stone-ČCh compactification of the positive integers. In this paper, we use mainly elementary functional analysis, but we will make some comments about the measure theoretic approach in Remarks 4.4 and 5.3 .

In Section 2, we define a skew linear operator, $A$, from a Banach space, $E$ into its dual and, in Theorem 2.2. we establish an upper bound for the quadratic form $-\left\langle A^{*} x^{* *}, x^{* *}\right\rangle$ on $E^{* *} \times E^{* *}$ under certain circumstances. See eqn. (2.3).

*Department of Mathematics, University of California, Santa Barbara, CA 93106-3080, U.S.A. Email: stesim38@gmail.com. 
In eqn. (3.1), we give the exact formula for $G$. Our presentation exploits the fact that $G$ can be "factorized through $c$ ". In Lemma 3.2 we discuss a particular element $x_{0}^{* *} \in \ell_{1}^{* *}=\ell_{\infty}^{*}$ and give formulae for $G^{*} x_{0}^{* *}$ and $\left\langle G^{*} x_{0}^{* *}, x_{0}^{* *}\right\rangle$. Lemma 3.3 appears in [5. Proposition, p. 360], but with a very different proof. Lemma 3.3 leads rapidly to our main result, Theorem 3.5

In Section 4 we give some technical results on $\ell_{1}, \ell_{1}^{*}, \ell_{1}^{* *}$ and $\ell_{1}^{* * *}$ and, in Theorem 4.2, define a particular element $w^{* * *}$ of $\ell_{1}^{* * *}$ that will be used in Section 5 to obtain formulae for $G^{*} x^{* *}$ and $\left\langle G^{*} x^{* *}, x^{* *}\right\rangle$ for general $x^{* *} \in \ell_{1}^{* *}$. It was proved in [4, Example, p. 89] and [1, Example 14.2.2, pp. 161-162] that, for all $x^{* *} \in \ell_{1}^{* *},\left\langle G^{*} x^{* *}, x^{* *}\right\rangle \leq 0$. In (5.2), we strengthen these results by showing that $\left\langle G^{*} x^{* *}, x^{* *}\right\rangle=-\left\langle x^{* *}, w^{* * *}\right\rangle^{2}$.

All Banach spaces considered in this note are real.

The author would like to express his thanks to Heinz Bauschke for reading the first draft of this paper and making a number of extremely valuable suggestions. He would also like to thank Jerry Beer for a very illuminating discussion on compactifications, which simplified considerably the analysis in Remark 4.4

\section{On skew linear operators on general Banach spaces}

Definition 2.1. Let $E$ be a nonzero Banach space and $A: E \rightarrow E^{*}$ be linear. We say that $A$ is skew if,

$$
\text { for all } w, x \in E,\langle w, A x\rangle=-\langle x, A w\rangle \text {, }
$$

or, equivalently,

$$
\text { for all } x \in E,\langle x, A x\rangle=0 .
$$

If $x \in E$, we write $\widehat{x}$ for the canonical image of $x$ in $E^{* *}$, that is to say $x \in E$ and $x^{*} \in E^{*} \Longrightarrow\left\langle x^{*}, \widehat{x}\right\rangle=\left\langle x, x^{*}\right\rangle$.

We recall that if $X$ and $Y$ are Banach spaces and $A: X \rightarrow Y$ is linear then the adjoint $A^{*}: Y^{*} \rightarrow X^{*}$ is defined by $\left\langle x, A^{*} y^{*}\right\rangle=\left\langle A x, y^{*}\right\rangle \quad(x \in X$, $\left.y^{*} \in Y^{*}\right)$.

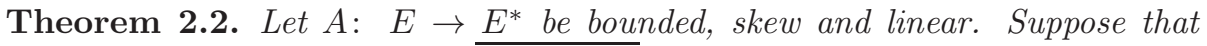
$x^{* *} \in E^{* *}, \lambda>0$ and $A^{*} x^{* *} \in \overline{R(A+\lambda J)}$. Then

$$
-\left\langle A^{*} x^{* *}, x^{* *}\right\rangle \leq \frac{1}{4} \lambda\left\|x^{* *}\right\|^{2} .
$$

Proof. Let $\varepsilon>0$. By hypothesis, there exist $x \in E, x^{*} \in E^{*}$ with

$$
\left\|x^{*}\right\|=\|x\|,\left\langle x, x^{*}\right\rangle=\|x\|^{2},
$$

and $z^{*} \in E^{*}$ such that $\left\|z^{*}\right\|<\varepsilon$ and $A^{*} x^{* *}=A x+\lambda x^{*}+z^{*}$. Then

$$
A^{*} x^{* *}-A x=\lambda x^{*}+z^{*},
$$


and, using (2.4),

$$
-\left\langle x^{*}, x^{* *}\right\rangle \leq\left\|x^{*}\right\|\left\|x^{* *}\right\|=\left\|x^{* *}\right\|\|x\| .
$$

Let $Z_{\varepsilon}:=\left\|x^{* *}\right\|+\varepsilon / \lambda$. From the definition of $A^{*}$, (2.2), (2.4)-(2.6), and the inequalities $\left\|z^{*}\right\|<\varepsilon$ and $-\|x\|^{2}+Z_{\varepsilon}\|x\| \leq \frac{1}{4} Z_{\varepsilon}^{2}$,

$$
\begin{aligned}
-\left\langle A^{*} x^{* *}, x^{* *}\right\rangle & =-\left\langle x, A^{*} x^{* *}-A x\right\rangle-\left\langle A^{*} x^{* *}-A x, x^{* *}\right\rangle \\
& =-\left\langle x, \lambda x^{*}+z^{*}\right\rangle-\left\langle\lambda x^{*}+z^{*}, x^{* *}\right\rangle \\
& \leq-\lambda\|x\|^{2}+\varepsilon\|x\|+\lambda\left\|x^{* *}\right\|\|x\|+\varepsilon\left\|x^{* *}\right\| \\
& =-\lambda\|x\|^{2}+\lambda Z_{\varepsilon}\|x\|+\varepsilon\left\|x^{* *}\right\| \leq \frac{1}{4} \lambda Z_{\varepsilon}^{2}+\varepsilon\left\|x^{* *}\right\| .
\end{aligned}
$$

Since $Z_{\varepsilon} \rightarrow\left\|x^{* *}\right\|$ as $\varepsilon \rightarrow 0$, (2.3) now follows by letting $\varepsilon \rightarrow 0$.

\section{Gossez's skew linear operator}

Definition 3.1. In the interest of precision, we shall use three different notations for the three duality pairings that appear in the rest of this paper. Then (noting that $\ell_{1}^{*}=\ell_{\infty}$ ), the bilinear form $\langle\cdot, \cdot\rangle_{0}: \ell_{1} \times \ell_{1}^{*} \rightarrow \mathbb{R}$ is defined in the usual way. Then $\ell_{1}^{* *}=\ell_{\infty}^{*}$, but this space does not have a convenient sequential representation. In this connection, see Remark 4.4. Also, $\langle\cdot, \cdot\rangle_{1}: \ell_{1}^{*} \times \ell_{1}^{* *} \rightarrow \mathbb{R}$ and $\langle\cdot, \cdot\rangle_{2}: \ell_{1}^{* *} \times \ell_{1}^{* * *} \rightarrow \mathbb{R}$. We write $\|\cdot\|_{1}$ and $\|\cdot\|_{\infty}$ for the usual norms on $\ell_{1}$ and $\ell_{\infty}$. Let $c$ be the subspace of $\ell_{\infty}$ consisting of all convergent sequences. Finally, let $e^{*}:=(1,1, \ldots) \in c$. In what follows, all sequences are indexed by the set $\{1,2,3, \ldots\}$.

Define the linear operator $G: \ell_{1} \rightarrow \ell_{1}^{*}=\ell_{\infty}$ by

$$
\text { for all } x \in \ell_{1},(G x)_{m}=\sum_{n>m} x_{n}-\sum_{n<m} x_{n} \text {. }
$$

$G$ is the "Gossez operator". It is well known that $G$ is skew and maximally monotone. See [4, Example, p. 89]. Clearly, for all $x \in \ell_{1}$,

$$
\lim _{m \rightarrow \infty}(G x)_{m}=-\sum_{n=1}^{\infty} x_{n}=-\left\langle x, e^{*}\right\rangle_{0} .
$$

Lemma 3.2. There exists $x_{0}^{* *} \in \ell_{1}^{* *}=\ell_{\infty}^{*}$ such that

$$
\begin{gathered}
\left\|x_{0}^{* *}\right\|=1, \\
G^{*} x_{0}^{* *}=-e^{*} \in \ell_{1}^{*}=\ell_{\infty}
\end{gathered}
$$

and

$$
\left\langle G^{*} x_{0}^{* *}, x_{0}^{* *}\right\rangle_{1}=-1 .
$$

Proof. The map from $c$ into $\mathbb{R}$ defined by $x^{*} \mapsto \lim _{m \rightarrow \infty} x_{m}^{*}$ is bounded and linear and has norm 1 on the vector subspace $c$ of $\ell_{\infty}$. So, from the extension form of the Hahn-Banach theorem, there exists $x_{0}^{* *} \in \ell_{\infty}^{*}$ such that (3.3) is satisfied and,

$$
\text { for all } x^{*} \in c,\left\langle x^{*}, x_{0}^{* *}\right\rangle_{1}=\lim _{m \rightarrow \infty} x_{m}^{*} \text {. }
$$


For all $x \in \ell_{1}, G x \in c$. Thus, from (3.2),

$$
\left\langle x, G^{*} x_{0}^{* *}\right\rangle_{0}=\left\langle G x, x_{0}^{* *}\right\rangle_{1}=\lim _{m \rightarrow \infty}(G x)_{m}=-\left\langle x, e^{*}\right\rangle_{0} .
$$

This completes the proof of (3.4). From this, $\left\langle G^{*} x_{0}^{* *}, x_{0}^{* *}\right\rangle_{1}=\left\langle-e^{*}, x_{0}^{* *}\right\rangle_{1}$ and (3.5) is immediate from (3.6).

The proof of Lemma 3.3 below is based on that of [5. Proposition, p. 360]. However, instead of using measure theory on $\beta \mathbb{N}$, we use the fact that a linear subspace is closed under differences (in (3.12) and (3.13)) and sums (in (3.14)). There is another way of establishing Lemma 3.3, using Rugged Banach spaces. See [1, Proposition 15.3.8, p. 176].

Lemma 3.3. Let $\lambda>0$. Suppose that

$$
\overline{R(G+\lambda J)} \text { is convex. }
$$

Then

$$
\overline{R(G+\lambda J)}=\ell_{\infty} .
$$

Proof. Let $k \geq 1$. As observed in [5. Proposition, p. 360], if, for all $m \notin\{1,2\}$, $\left|u_{m}^{*}\right| \leq 2 \lambda k$, then

$$
\left(-k+2 \lambda k,-k-2 \lambda k, u_{3}^{*}, u_{4}^{*}, u_{5}^{*}, \ldots\right) \in(G+\lambda J)\left(k e_{1}-k e_{2}\right) \in \overline{R(G+\lambda J)} .
$$

In particular,

$$
(-k+2 \lambda k,-k-2 \lambda k, 0,0,0, \ldots) \in \overline{R(G+\lambda J)} .
$$

As observed in [5, Proposition, p. 360], (3.8) implies that $\overline{R(G+\lambda J)}$ is a linear subspace of $\ell_{\infty}$. So, by subtracting (3.11) from (3.10),

$$
\left(0,0, u_{3}^{*}, u_{4}^{*}, u_{5}^{*}, \ldots\right) \in \overline{R(G+\lambda J)} .
$$

Similarly, if, for all $m \notin\{3,4\},\left|v_{m}^{*}\right| \leq 2 \lambda k$, then

$$
\left(v_{1}^{*}, v_{2}^{*}, 0,0, v_{5}^{*}, \ldots\right) \in \overline{R(G+\lambda J)} .
$$

Taking the Minkowski sum of (3.12) and (3.13),

$$
\left(v_{1}^{*}, v_{2}^{*}, u_{3}^{*}, u_{4}^{*}, u_{5}^{*}+v_{5}^{*}, u_{6}^{*}+v_{6}^{*}, \ldots\right) \in \overline{R(G+\lambda J)} .
$$

(3.9) now follows easily by letting $k \rightarrow \infty$.

Lemma 3.4. Suppose that $\lambda>0$ and $\overline{R(G+\lambda J)}$ is convex. Then $\lambda \geq 4$.

Proof. Let $x_{0}^{* *}$ be as in Lemma 3.2 From Lemma 3.3. $G^{*} x_{0}^{* *} \in \overline{R(G+\lambda J)}$. From Theorem 2.2, (3.5) and (3.3), $1=-\left\langle G^{*} x_{0}^{* *}, x_{0}^{* *}\right\rangle \leq \frac{1}{4} \lambda\left\|x_{0}^{* *}\right\|^{2}=\frac{1}{4} \lambda$. This gives the desired result.

Theorem 3.5. If $0<\lambda<4$ then $\overline{R(G+\lambda J)}$ is not convex. In particular, $\overline{R(G+J)}$ is not convex.

Proof. This is immediate from Lemma 3.4.

Problem 3.6. Is $\overline{R(G+4 J)}$ convex? 


\section{On the dual, bidual and tridual of $\ell_{1}$}

This section is devoted to the technical results that will be needed for our discussion of $G^{*}$ in Section 5. We point, in particular, to Lemma 4.1(c), in which $p^{*}$ is moved from being the first variable in $\langle\cdot, \cdot\rangle_{1}$ to being the second variable in $\langle\cdot, \cdot\rangle_{0}$, i.e., from being a primal variable to being a dual variable. Lemma 4.1(c) will be critical in the proof of (4.3), which will be used in Theorem 5.1.

Let $c_{0}$ be the Banach space of sequences that converge to 0 . For all $m \geq 1$, let $e_{m}^{*}$ be the element $(0, \ldots, 0,1,0,0, \ldots)$ of $\ell_{1}^{*}$, with the 1 in the $m$ th place. Define the linear map $W: \ell_{1}^{* *} \rightarrow \ell_{1}$ by $W x^{* *}:=\left(\left\langle e_{m}^{*}, x^{* *}\right\rangle_{1}\right)_{m \geq 1}$.

Lemma 4.1. (a) Let $x^{* *} \in \ell_{1}^{* *}$. Then $\sum_{m=1}^{\infty}\left|\left\langle e_{m}^{*}, x^{* *}\right\rangle_{1}\right| \leq\left\|x^{* *}\right\|<\infty$.

(b) $\|W\|=1$ and, for all $x \in \ell_{1}, W \widehat{x}=x$.

(c) Let $p^{*} \in c_{0} \subset \ell_{1}^{*}$ and $x^{* *} \in \ell_{1}^{* *}$. Then $\left\langle p^{*}, x^{* *}\right\rangle_{1}=\left\langle W x^{* *}, p^{*}\right\rangle_{0}$.

Proof. For all $m \geq 1$, find $\delta_{m}$ such that $\left|\delta_{m}\right|=1$ and $\delta_{m}\left\langle e_{m}^{*}, x^{* *}\right\rangle_{1}=\left|\left\langle e_{m}^{*}, x^{* *}\right\rangle_{1}\right|$. Let $n \geq 1$. Then

$$
\begin{aligned}
\sum_{m=1}^{n}\left|\left\langle e_{m}^{*}, x^{* *}\right\rangle_{1}\right| & =\sum_{m=1}^{n} \delta_{m}\left\langle e_{m}^{*}, x^{* *}\right\rangle_{1}=\left\langle\sum_{m=1}^{n} \delta_{m} e_{m}^{*}, x^{* *}\right\rangle_{1} \\
& \leq\left\|\sum_{m=1}^{n} \delta_{m} e_{m}^{*}\right\|_{\infty}\left\|x^{* *}\right\|=\sup _{m=1}^{n}\left|\delta_{m}\right|\left\|x^{* *}\right\|=\left\|x^{* *}\right\| .
\end{aligned}
$$

(a) now follows by letting $n \rightarrow \infty$. It also follows that $\left\|W x^{* *}\right\|_{1} \leq\left\|x^{* *}\right\|$. Since this holds for all $x^{* *} \in \ell_{1}^{* *},\|W\| \leq 1$. Now let $x \in \ell_{1}$. Then, for all $m \geq 1$, $(W \widehat{x})_{m}=\left\langle e_{m}^{*}, \widehat{x}\right\rangle_{1}=\left\langle x, e_{m}^{*}\right\rangle_{0}=x_{m}$, and so $W \widehat{x}=x$, as required. It follows from this that $\|W\|=1$, which completes the proof of (b).

Let $p^{*}=\left(p_{m}\right)_{m \geq 1} \in c_{0}$. Since $p^{*}=\lim _{n \rightarrow \infty} \sum_{m=1}^{n} p_{m} e_{m}^{*}$ in $\ell_{\infty}$,

$$
\begin{aligned}
\left\langle p^{*}, x^{* *}\right\rangle_{1} & =\lim _{n \rightarrow \infty} \sum_{m=1}^{n} p_{m}\left\langle e_{m}^{*}, x^{* *}\right\rangle_{1}=\sum_{m=1}^{\infty} p_{m}\left\langle e_{m}^{*}, x^{* *}\right\rangle_{1} \\
& =\sum_{m=1}^{\infty}\left\langle e_{m}^{*}, x^{* *}\right\rangle_{1} p_{m}=\left\langle W x^{* *}, p^{*}\right\rangle_{0} .
\end{aligned}
$$

This completes the proof of $(\mathrm{c})$.

In what follows, we define $w^{* * *}:=\widehat{e^{*}}-W^{*} e^{*} \in \ell_{1}^{* * *}$.

Theorem 4.2. We have

$$
\left\|w^{* * *}\right\|=1
$$

for all $x \in \ell_{1}$,

$$
\left\langle\widehat{x}, w^{* * *}\right\rangle_{2}=0,
$$

and, for all $x^{*}=\left(x_{m}^{*}\right)_{m \geq 1} \in c$ and $x^{* *} \in \ell_{1}^{* *}$,

$$
\left\langle x^{*}, x^{* *}\right\rangle_{1}=\left\langle W x^{* *}, x^{*}\right\rangle_{0}+\left\langle x^{* *}, w^{* * *}\right\rangle_{2} \lim _{n \rightarrow \infty} x_{n}^{*} .
$$

Proof. Let $n \geq 1$. Then

$$
\begin{aligned}
\left\langle e^{*}, x^{* *}\right\rangle_{1}-\sum_{m=1}^{n}\left\langle e_{m}^{*}, x^{* *}\right\rangle_{1} & =\left\langle e^{*}-\sum_{m=1}^{n} e_{m}^{*}, x^{* *}\right\rangle_{1} \\
& \leq\left\|e^{*}-\sum_{m=1}^{n} e_{m}^{*}\right\|_{\infty}\left\|x^{* *}\right\|=\left\|x^{* *}\right\| .
\end{aligned}
$$


Letting $n \rightarrow \infty,\left\langle e^{*}, x^{* *}\right\rangle_{1}-\sum_{m=1}^{\infty}\left\langle e_{m}^{*}, x^{* *}\right\rangle_{1} \leq\left\|x^{* *}\right\|$. Thus

$$
\begin{aligned}
\left\langle x^{* *}, w^{* * *}\right\rangle_{2} & =\left\langle x^{* *}, \widehat{e}^{*}\right\rangle_{2}-\left\langle x^{* *}, W^{*} e^{*}\right\rangle_{2}=\left\langle e^{*}, x^{* *}\right\rangle_{1}-\left\langle W x^{* *}, e^{*}\right\rangle_{0} \\
& =\left\langle e^{*}, x^{* *}\right\rangle_{1}-\sum_{m=1}^{\infty}\left\langle e_{m}^{*}, x^{* *}\right\rangle_{1} \leq\left\|x^{* *}\right\| .
\end{aligned}
$$

Since this holds for all $x^{* *} \in \ell_{1}^{* *},\left\|w^{* * *}\right\| \leq 1$. On the other hand, if $x_{0}^{* *}$ is as in Lemma 3.2, then (3.3) gives $\left\|x_{0}^{* *}\right\|=1$ and, from (3.6) and the above,

$$
\left\langle x_{0}^{* *}, w^{* * *}\right\rangle_{2}=\left\langle e^{*}, x_{0}^{* *}\right\rangle_{1}-\sum_{m=1}^{\infty}\left\langle e_{m}^{*}, x_{0}^{* *}\right\rangle_{1}=1-\sum_{m=1}^{\infty} 0=1,
$$

which gives (4.1). Now let $x \in \ell_{1}$. Then, from Lemma 4.1(b),

$$
\begin{aligned}
\left\langle\widehat{x}, w^{* * *}\right\rangle_{2} & =\left\langle\widehat{x}, \widehat{e^{*}}\right\rangle_{2}-\left\langle\widehat{x}, W^{*} e^{*}\right\rangle_{2}=\left\langle e^{*}, \widehat{x}\right\rangle_{1}-\left\langle W \widehat{x}, e^{*}\right\rangle_{0} \\
& =\left\langle x, e^{*}\right\rangle_{0}-\left\langle x, e^{*}\right\rangle_{0}=0
\end{aligned}
$$

which gives (4.2). Finally, let $x^{*}=\left(x_{m}^{*}\right)_{m \geq 1} \in c, x^{* *} \in \ell_{1}^{* *}$, and write $\Lambda:=$ $\lim _{n \rightarrow \infty} x_{n}^{*}$. From Lemma 4.1(c), with $p^{*}:=x^{*}-\Lambda e^{*} \in c_{0}$,

$$
\left\langle x^{*}-\Lambda e^{*}, x^{* *}\right\rangle_{1}=\left\langle W x^{* *}, x^{*}-\Lambda e^{*}\right\rangle_{0}
$$

Thus

$$
\begin{aligned}
\left\langle x^{*}, x^{* *}\right\rangle_{1} & =\left\langle W x^{* *}, x^{*}-\Lambda e^{*}\right\rangle_{0}+\left\langle\Lambda e^{*}, x^{* *}\right\rangle_{1} \\
& =\left\langle W x^{* *}, x^{*}\right\rangle_{0}-\left\langle W x^{* *}, \Lambda e^{*}\right\rangle_{0}+\left\langle\Lambda e^{*}, x^{* *}\right\rangle_{1},
\end{aligned}
$$

which gives (4.3). This completes the proof of Theorem 4.2

Remark 4.3. For all $x^{* *} \in \ell_{1}^{* *}=\ell_{\infty}^{*},\left\langle x^{* *}, W^{*} e^{*}\right\rangle_{2}=\left\langle W x^{* *}, e^{*}\right\rangle_{0}=$ $\sum_{m=1}^{\infty}\left\langle e_{m}^{*}, x^{* *}\right\rangle_{1}=\sum_{m=1}^{\infty}\left\langle x^{* *}, \widehat{e_{m}^{*}}\right\rangle_{2}$, so we could write $W^{*} e^{*}=\sum_{m=1}^{\infty} \widehat{e_{m}^{*}}$, with the understanding that the convergence is in the $w\left(\ell_{1}^{* * *}, \ell_{1}^{* *}\right)$ (weak ${ }^{*}$ ) sense. Thus, with this understanding, $w^{* * *}=\widehat{e^{*}}-\sum_{m=1}^{\infty} \widehat{e_{m}^{*}}$. Note from (4.1) that this does not imply that $w^{* * *}=0$.

Since $\widehat{W \widehat{x}}=\widehat{x}$, the map $x^{* *} \mapsto \widehat{W x^{* *}}$ is a linear retraction from $\ell_{1}^{* *}$ onto $\widehat{\ell}_{1}$.

Remark 4.4. It is known from standard results in point-set topology that the set $\mathbb{N}$ of positive integers (considered as a discrete topological space) can be embedded as a dense open subspace of a compact Hausdorff space, $\beta \mathbb{N}$ (the Stone- $\breve{C e c h}$ compactification of $\mathbb{N}$ ), such that, for all $x^{*} \in \ell_{1}^{*}=\ell_{\infty}$, there exists a unique element $\beta x^{*}$ of $C(\beta \mathbb{N})$ (the set of continuous functions on $\beta \mathbb{N}$ ) extending $x^{*}$. (The fact that $\mathbb{N}$ is open in $\beta \mathbb{N}$ is a consequence of the result proved in [2, XI.8.3, pp. 245-246] that any locally compact completely regular space is open in any compactification.) Obviously $\beta e^{*}=1$.

For all $m \geq 1,\{m\}$ is open (in $\mathbb{N}$ and hence) in $\beta \mathbb{N}$, and so, if $f_{m}: \beta \mathbb{N} \rightarrow \mathbb{R}$ is defined by $f_{m}(m):=1$ and $f_{m}:=0$ on $\beta \mathbb{N} \backslash\{m\}$ then $f_{m}=\beta e_{m}^{*} \in C(\beta \mathbb{N})$. It follows from the Riesz representation theorem (see, for instance, 6, Theorem 6.19 , pp. $130-132]$ for a considerably more general result) that $\ell_{1}^{* *}=\ell_{\infty}^{*}$ can be identified with the set $\mathcal{M}(\beta \mathbb{N})$ of (signed) Radon measures on $\beta \mathbb{N}$. If $x^{* *} \in$ 
$\ell_{1}^{* *}=\ell_{\infty}^{*}$ and $\mu \in \mathcal{M}(\beta \mathbb{N})$ represents $x^{* *}$ then $\left\langle e_{m}^{*}, x^{* *}\right\rangle_{1}=\int f_{m} d \mu=\mu(\{m\})$, and so $W x^{* *}=(\mu(\{m\}))_{m \geq 1} \in \ell_{1}$. Furthermore, $\left\langle e^{*}, x^{* *}\right\rangle_{1}=\int 1 d \mu=\mu(\beta \mathbb{N})$. Thus, from (4.4) and standard measure-theoretic arguments,

$$
\left\langle x^{* *}, w^{* * *}\right\rangle_{2}=\mu(\beta \mathbb{N})-\sum_{m=1}^{\infty} \mu(\{m\})=\mu(\beta \mathbb{N} \backslash \mathbb{N}) .
$$

This discussion will be continued in Remark 5.3 .

\section{$5 G^{*}$}

Theorem 5.1 below extends the results proved in (3.4) and (3.5) for a particular element $x_{0}^{* *}$ of $\ell_{1}^{* *}$ to a general element $x^{* *}$ of $\ell_{1}^{* *}$.

Theorem 5.1. Let $x^{* *} \in \ell_{1}^{* *}$. Then

$$
G^{*} x^{* *}=-G W x^{* *}-\left\langle x^{* *}, w^{* * *}\right\rangle_{2} e^{*} \in \ell_{1}^{*}
$$

and

$$
\left\langle G^{*} x^{* *}, x^{* *}\right\rangle_{1}=-\left\langle x^{* *}, w^{* * *}\right\rangle_{2}^{2} .
$$

Proof. Let $x \in \ell_{1}$. Setting $x^{*}=G x$ in (4.3), writing $\alpha$ for $\left\langle x^{* *}, w^{* * *}\right\rangle_{2}$ to simplify the expressions, and using (2.1) (with $w=W x^{* *}$ ) and (3.2),

$$
\begin{aligned}
\left\langle x, G^{*} x^{* *}\right\rangle_{0} & =\left\langle G x, x^{* *}\right\rangle_{1}=\left\langle W x^{* *}, G x\right\rangle_{0}+\alpha \lim _{n \rightarrow \infty}(G x)_{n} \\
& =-\left\langle x, G W x^{* *}\right\rangle_{0}-\alpha\left\langle x, e^{*}\right\rangle_{0}
\end{aligned}
$$

Since this holds for all $x \in \ell_{1}$, this completes the proof of (5.1). From (5.1), the definition of $G^{*}$, (5.1) again, (2.2) (with $x=W x^{* *}$ ), the definition of $W^{*}$, and the definition of $w^{* * *}$ (in sequence),

$$
\begin{aligned}
\left\langle G^{*} x^{* *}, x^{* *}\right\rangle_{1} & =-\left\langle G W x^{* *}, x^{* *}\right\rangle_{1}-\alpha\left\langle e^{*}, x^{* *}\right\rangle_{1} \\
& =-\left\langle W x^{* *}, G^{*} x^{* *}\right\rangle_{0}-\alpha\left\langle e^{*}, x^{* *}\right\rangle_{1} \\
& =\left\langle W x^{* *}, G W x^{* *}\right\rangle_{0}+\alpha\left\langle W x^{* *}, e^{*}\right\rangle_{0}-\alpha\left\langle e^{*}, x^{* *}\right\rangle_{1} \\
& =\alpha\left\langle W x^{* *}, e^{*}\right\rangle_{0}-\alpha\left\langle e^{*}, x^{* *}\right\rangle_{1} \\
& =\alpha\left[\left\langle x^{* *}, W^{*} e^{*}\right\rangle_{2}-\left\langle x^{* *}, e^{*}\right\rangle_{2}\right]=-\alpha^{2} .
\end{aligned}
$$

This gives (5.2), and completes the proof of Theorem 5.1.

Remark 5.2. There is an analysis of $G^{*}$ and $\left\langle G^{*} x^{* *}, x^{* *}\right\rangle_{1}$ in 1, Example 14.2.2, pp. 161-162] that is, on the surface, different from the one presented in Theorem 5.1 above. A linear operator $T: \ell_{1} \rightarrow \ell_{\infty}$ is defined by

$$
\text { for all } x \in \ell_{1},(T x)_{m}=x_{m}+2 \sum_{n>m} x_{n} \text {. }
$$

If $n$ is odd, we define $y^{(n)} \in \ell_{1}$ by $y^{(n)}:=(2,-2, \ldots, 2,-2,1,0,0, \ldots)$, where the " 1 " is in the $n$th place. Then

$$
T\left(y^{(n)}\right)=(2,-2, \ldots, 2,-2,1,0,0, \ldots)+2(-1,1, \ldots,-1,1,0,0,0, \ldots)=e_{n}^{*} .
$$


Similarly, if $n$ is even, we define $y^{(n)} \in \ell_{1}$ by $y^{(n)}:=(-2,2, \ldots, 2,-2,1,0,0, \ldots)$. Again, $T\left(y^{(n)}\right)=e_{n}^{*}$. The analysis in 1] rests on the assumption (see [1, Eqn. (1), pp. 159]) that, for all $x^{* *} \in \ell_{1}^{* *}$,

there exists $x \in \ell_{1}$ such that, for all $x^{*} \in R(T),\left\langle x^{*}, \widehat{x}\right\rangle_{1}=\left\langle x^{*}, x^{* *}\right\rangle_{1}$.

In particular, the argument above implies that, for all $n \geq 1,\left\langle e_{n}^{*}, \widehat{x}\right\rangle_{1}=$ $\left\langle e_{n}^{*}, x^{* *}\right\rangle_{1}$. Thus, for all $n \geq 1, x_{n}=\left\langle e_{n}^{*}, x^{* *}\right\rangle_{1}$. Consequently, $x=W x^{* *}$, and the formulae for $G^{*}$ and $\left\langle G^{*} x^{* *}, x^{* *}\right\rangle_{1}$ given in [1] reduce to the more explicit ones given in Theorem [5.1] above.

Remark 5.3. This is a continuation of Remark 4.4 A comparison of (4.5) and (5.2) leads to the conclusion that $\left\langle G^{*} x^{* *}, x^{* *}\right\rangle_{1}=-\mu(\beta \mathbb{N} \backslash \mathbb{N})^{2}$. This is exactly the formula obtained in Gossez, 4, Example, p. 89].

\section{References}

[1] H. H. Bauschke, Projection Algorithms and Monotone Operators, http://summit.sfu.ca/item/7015.

[2] J. Dugundji, Topology, Allyn and Bacon, Inc., Boston-London-Sydney, 1978.

[3] J.-P. Gossez, Opérateurs monotones non linéaires dans les espaces de Banach non réflexifs, J. Math. Anal. Appl. 34 (1971), 371-395.

[4] J.-P. Gossez, On the range of a coercive maximal monotone operator in a nonreflexive Banach space, Proc. Amer. Math. Soc. 35 (1972), 88-92.

[5] J.-P. Gossez, On a convexity property of the range of a maximal monotone operator, Proc. Amer. Math. Soc. 55 (1976), 359-360.

[6] W. Rudin, Real and complex analysis, Third edition, McGraw-Hill Book Co., New York, 1987. 